\title{
Proposed risk factors for infection with multidrug-resistant pathogens in hemodialysis patients hospitalized with pneumonia
}

\author{
Jae-Uk Song ${ }^{1+}$, Hye Kyeong Park ${ }^{2+}$, Hyung Koo Kang ${ }^{2}$ and Jonghoo Lee (D) $^{3^{*+}}$
}

\begin{abstract}
Background: In patients with hemodialysis-associated pneumonia (HDAP), information on both microbiologic features and antimicrobial strategies is limited. The aim of this study is to investigate predictive factors of infection with multidrug-resistant (MDR) pathogens in HDAP patients.

Methods: This was a multicenter, retrospective, and observational study. Enrolled patients were classified into MDR or non-MDR pathogens groups according to culture results. We examined risk factors of infection with MDR pathogens and created a decision support tool using these risk factors.

Results: MDR pathogens were identified in 24 (22.8\%) out of a total of 105 HDAP patients. The most common MDR pathogens were methicillin-resistant Staphylococcus aureus (10 patients, 9.5\%) and the isolation rate of Pseudomonas aeruginosa was 6.6\%. Logistic regression showed two variables were associated with the isolation of MDR pathogens: recent hospitalization (adjusted odds ratio [OR]: 2.951, 95\% confidence interval [CI]: 1.022-8.518) and PSI (Pneumonia Severity Index) score (adjusted OR: 1.023, 95\% Cl: 1.005-1.041). The optimal cut-off value for PSI score using a receiver operating characteristic curve analysis was 147. According to the presence of 0,1 , or 2 of the identified risk factors, the prevalence of MDR pathogens was 7.6, 28.2 and 64.2\%, respectively ( $p<0.001$ for trend). The area under the curve of the prediction tool was 0.764 (95\% Cl: 0.652-0.875).

Conclusions: We demonstrated that recent hospitalization and PSI > 147 are risk factors of infection with MDR pathogens in HDAP patients. This simple proposed tool would facilitate more accurate identification of MDR pathogens in these patients.
\end{abstract}

Keywords: Pneumonia, Hemodialysis, End-stage renal disease, Multidrug resistance, Pathogen

\section{Background}

In hemodialysis (HD) populations, pneumonia is common and a leading cause of death $[1,2]$. Because of the uremic internal milieu and the very frequent coexistence of serious comorbid medical conditions, these patients can be considered chronically immunosuppressed [3]. According to the United States Renal Dada System (USRDS) registry, approximately $20 \%$ of patients developed pneumonia in

\footnotetext{
* Correspondence: lovlet@paran.com

${ }^{\dagger}$ Equal contributors

${ }^{3}$ Department of Internal Medicine, Jeju National University Hospital, Jeju National University School of Medicine, Aran 13 gil 15, Jeju-si, Jeju Specia Self-Governing Province 690-767, South Korea

Full list of author information is available at the end of the article
}

the 1-year period following initiation of dialysis therapy [1]. The mortality rates from pneumonia in hemodialysis (HD) patients were higher than those from pneumonia in the general population [2]. Therefore, early proper management is important to reduce mortality in HD patients with pneumonia.

Until now, there are no guidelines focused primarily on hemodialysis-associated pneumonia (HDAP). Because the 2005 American Thoracic Society (ATS)/Infectious Diseases Society of America (IDSA) guidelines included HDAP as a category of HCAP, HDAP patients could receive broadspectrum antibiotics targeted against multidrug-resistant (MDR) pathogens [4]. But, several studies demonstrated 
that HCAP does not always identify MDR pathogens [5]. The 2016 ATS/IDSA guidelines removed the concept of HCAP among a category of nosocomial pneumonia [6].

The clinical epidemiology of HDAP has received little attention to date [7]. A previous study including some data used in the present study revealed that the HDAP group was clinically more similar to the CAP group than to the HCAP other than HDAP (O-HCAP) [7]. Accordingly, whether MDR pathogens-targeted antibiotics should be selected in patients with HDAP is unclear. Because of the uncertainty surrounding the actual risks of infection with MDR pathogens in HDAP patients and the increasing burden of end-stage renal disease worldwide [8], more data are required for a better distinct targeted therapeutic approach. Therefore, we investigated microbiologic characteristics and novel predictive factors of infection with MDR pathogens in patients hospitalized with HDAP. We also developed a prediction tool using these risk factors to identify subjects infected with MDR pathogens.

\section{Methods}

\section{Study design, populations, and recorded parameters}

We retrospectively conducted observational cohort studies at three institutions (Jeju National University Hospital, Kangbuk Samsung Hospital, and Ilsan Paik Hospital) between January 2011 and December 2015. Some of the clinical data for patients enrolled at Jeju National University Hospital were included in an article published in 2016 [7].

Patients were screened by the Korean Standard Classification of Diseases-7 codes of the followings; J18.0-18.9 as representative codes of pneumonia in the primary discharge diagnosis and N18.5, N18.9, or Z49.1 as codes of HD [9]. The medical records and radiological findings were reviewed to confirm the diagnosis of pneumonia by the following criteria: the presence of a new infiltrate on chest radiography with symptoms and signs of a lower respiratory tract infection. And patients on regular intermittent HD 3 times a week were included in the analysis. We excluded the following types of patients: (1) those who did not receive dialysis at the time of admission, (2) those who underwent continuous renal replacement therapy after organ failure developed by pneumonia, (3) those who had hospital-acquired pneumonia (HAP) developed at least $48 \mathrm{~h}$ after hospital admission, (4) those who did receive continuous ambulatory peritoneal dialysis, (5) those who re-visited within 10 days of discharging, and (6) those who transferred from other hospitals after hospitalization for $>48 \mathrm{~h}$.

According to culture results, enrolled patients were classified as MDR or non-MDR pathogens groups. We compared clinical characteristics, severity of pneumonia, identified pathogens, antibiotics, and clinical outcomes between the two groups. The severity of pneumonia was assessed by the CURB-65 (confusion, urea, respiratory rate, blood pressure, age more than 65 years) and Pneumonia Severity Index (PSI) scores [10, 11].

\section{Definitions}

HDAP was defined as pneumonia developing in patients receiving chronic HD within 30 days. O-HCAP was defined using the criteria of the 2005 ATS/IDSA guidelines as follows: recent history of hospitalization in an acute care hospital for $\geq 2$ days in the past 90 days; residence in a nursing home or long-term care facility (NHAP, nursing home-acquired pneumonia); or recent outpatient intravenous therapy or wound care within the past 30 days [4]. Severe pneumonia was defined according to ATS/IDSA 2007 criteria [12].

In accordance with the 2005 ATS/IDSA guidelines [4], methicillin-resistant Staphylococcus aureus (MRSA), Pseudomonas aeruginosa, extended-spectrum betalactamase (ESBL)-producing or carbapenem-resistant Klebsiella pneumoniae and Escherichia coli, Acinetobacter baumanii, and Stenotrophomonas maltophilia were considered to be MDR pathogens. According to susceptibility test criteria for lower respiratory tract pathogens, the appropriateness of antibiotic therapy was analyzed for all cases with an etiological diagnosis. Inappropriate antibiotic therapy was defined if the empirical antibiotics were not effective or unnecessarily broad against the identified pathogens based on in vitro susceptibility testing [13]. Failure of initial antibiotics therapy was defined as death during initial treatment or change of antibiotics from initial agents to others after $48 \mathrm{~h}$ due to clinical instability [14].

\section{Microbiology}

Sputum, tracheal aspirate, bronchial alveolar lavage fluid or blood samples were investigated for microbial analysis. Respiratory samples were cultured in a semi-quantitative manner, and pathogens were identified when a predominant microorganism was detected from group 4 or 5 sputum, according to Geckler's grading system [15]. Blood cultures were considered as pathogens if there was no other infection source for a positive blood culture. Paired serology for Mycoplasma pneumoniae or Chlamydia pneumoniae and urinary antigen tests for Streptococcus pneumoniae and Legionella pneumophila serogroup 1 were also recorded if these exams were checked. The antibiotic sensitivity of all isolates was determined using a disc diffusion method, according to the Clinical and Laboratory Standards Institute guidelines [16].

\section{Statistical analyses}

Data are presented as medians and interquartile ranges (IQRs; 25th and 75th percentiles) for continuous variables and as numbers and percentages for categorical variables. For comparison of continuous variables, the Mann-Whitney U-test between two 
groups and the Kruskal-Wallis test among three groups were used to compare the median values. Categorical variables were compared using the Pearson $\mathrm{X}^{2}$ test, and the Fisher's exact test was used when any cell contained fewer than five data points.

To identify independent predictive factors associated with occurrence of MDR pathogens, we performed multivariate logistic regression analyses, as measured by the estimated odds ratio (OR) with $95 \%$ confidence interval $(\mathrm{CI})$. Potential candidate variables with a $P$ value less than 0.05 in univariate analysis were entered into the regression model. From logistic regression results, we created predictive tool to identify patients with HDAP due to MDR pathogens. We classified patients based on the presence of risk factors for MDR pathogens. Then, we evaluated the predictive value of the proposed support tool for correctly indicating the presence of infection with MDR pathogens via a receiver operating characteristic (ROC) curve. The estimated area under the ROC curve (AUC) values were compared using the HanleyMcNeil test [17]. The cut-off point that showed the highest Youden Index was considered the optimal cut-off value [18]. All tests were two-sided, and $P$ values $<0.05$ were considered statistically significant. All analyses were performed using the Statistical Package for the Social Sciences (SPSS) network version 18.0 (SPSS; Chicago, IL, USA).

\section{Results}

Baseline characteristics and clinical outcomes

A total of 887 patients were initially identified through medical records. Of these patients, 703 were excluded for the reasons in the followings; 536 did not meet pneumonia definitions, 111 did not receive dialysis at the time of admission, and 56 underwent CCRT after organ failure developed by pneumonia (Fig. 1). A total of 184 patients (21.1\%) were enrolled as the dialysis related to pneumonia. Seventy-nine patients were excluded for the reasons presented in Fig. 1. Finally, a total of 105 patients were included in the present study.

There were 68 males and 37 females, with a median age of 71 years. MDR pathogens were identified in 24 patients (22.8\%). The baseline clinical characteristics and clinical outcomes of the total 105 HDAP patients are summarized in Table 1.

\section{Microbiological etiology}

Table 2 shows the distribution of causative organisms. Of the total 105 HDAP patients, the responsible pathogens were determined only in 53 patients (50.4\%). The most common pathogen was $S$. aureus (17, $16.1 \%)$, which consisted of methicillin-sensitive $S$. aureus $(7,6.6 \%)$ and MRSA (10, 9.5\%), followed by $K$. pneumoniae $(11,10.4 \%)$ and S. pneumoniae (10, $9.5 \%)$. The isolation rates of drug-resistant gram-

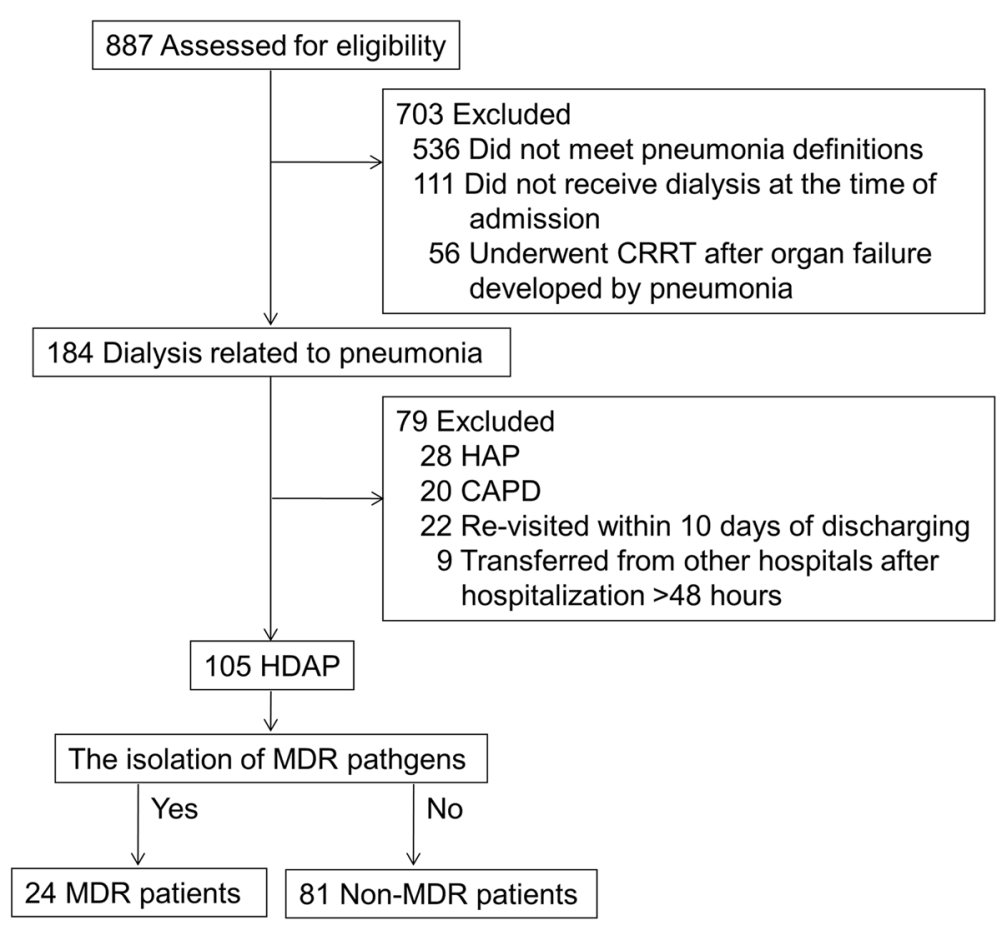

Fig. 1 Patient flow. CRRT, continuous renal replacement therapy; HAP, hospital-acquired pneumonia; CAPD, continuous ambulatory peritoneal dialysis; HDAP, hemodialysis-associated pneumonia; MDR, multidrug-resistant 
Table 1 Baseline clinical characteristics and treatment outcomes of patients admitted with hemodialysis-associated pneumonia

\begin{tabular}{|c|c|c|c|c|}
\hline Characteristics & $\begin{array}{l}\text { Overall patients } \\
(n=105)\end{array}$ & $\begin{array}{l}\text { MDR pathogens group } \\
(n=24)\end{array}$ & $\begin{array}{l}\text { Non-MDR pathogens group } \\
(n=81)\end{array}$ & $P$ Value \\
\hline Age (years) & $71(61-76)$ & $73(62-79)$ & $71(61-76)$ & 0.199 \\
\hline Male & $68(64.8 \%)$ & $15(62.5 \%)$ & $53(65.4 \%)$ & 0.792 \\
\hline Female & $37(35.2 \%)$ & $9(37.5)$ & $28(34.6 \%)$ & 0.792 \\
\hline $\begin{array}{l}\text { Time interval between dialysis and pneumonia } \\
\text { (months) }\end{array}$ & $30(11-69)$ & $21(10-74)$ & $34(12-69)$ & 0.541 \\
\hline \multicolumn{5}{|l|}{ Etiology of dialysis ${ }^{a}$} \\
\hline Diabetes mellitus & $60(57.1 \%)$ & $14(58.3 \%)$ & $46(56.7 \%)$ & 0.344 \\
\hline Hypertension & $57(54.2 \%)$ & $11(45.8 \%)$ & $46(56.7 \%)$ & 0.893 \\
\hline Glomerulonephropathy & $6(5.7 \%)$ & $1(4.1 \%)$ & $5(6.1 \%)$ & 1.000 \\
\hline Idiopathic & $7(6.6 \%)$ & $2(8.3 \%)$ & $5(6.1 \%)$ & 0.658 \\
\hline Others & $13(12.3 \%)$ & $1(4.1 \%)$ & $12(14.8 \%)$ & 0.289 \\
\hline Tube feeding & $9(8.5 \%)$ & $5(20.8 \%)$ & $4(4.9 \%)$ & 0.028 \\
\hline HCAP criteria other than HDAP & & $17(70.8 \%)$ & $27(33.3 \%)$ & 0.001 \\
\hline Recent hospitalization & $42(40.0 \%)$ & $16(66.6 \%)$ & $26(32.0 \%)$ & 0.002 \\
\hline NHAP & $10(9.5 \%)$ & $5(20.8 \%)$ & $5(6.1 \%)$ & 0.047 \\
\hline Recent intravenous therapy & $10(9.5 \%)$ & $3(12.5 \%)$ & $7(8.6 \%)$ & 0.692 \\
\hline \multicolumn{5}{|l|}{ Clinical parameters } \\
\hline Severe pneumonia & $37(35.2 \%)$ & $13(54.1 \%)$ & $24(29.6 \%)$ & 0.027 \\
\hline Confusion & $15(14.2 \%)$ & $6(25.0 \%)$ & $9(11.1 \%)$ & 0.103 \\
\hline Respiratory failure & $47(44.7 \%)$ & $14(58.3 \%)$ & $33(40.7 \%)$ & 0.128 \\
\hline Sepsis or septic shock at onset & $15(14.2 \%)$ & 7 (29.1\%) & $8(9.8 \%)$ & 0.040 \\
\hline ICU admission & $22(20.9 \%)$ & $10(41.6 \%)$ & $12(14.8 \%)$ & 0.005 \\
\hline Need for ventilator & $6(5.7 \%)$ & $3(12.5 \%)$ & $3(3.7 \%)$ & 0.131 \\
\hline \multicolumn{5}{|l|}{ Radiological findings } \\
\hline Multi-lobar involvement & $73(69.5 \%)$ & $20(83.3 \%)$ & $53(65.4 \%)$ & 0.094 \\
\hline Pleural effusion & $33(31.4 \%)$ & $6(25.0 \%)$ & $27(33.3 \%)$ & 0.440 \\
\hline \multicolumn{5}{|l|}{ Laboratory findings } \\
\hline WBC $\left(/ \mathrm{mm}^{3}\right)$ & $11,200(7400-15,015)$ & $13,210(8200-18,600)$ & $10,680(7150-14,960)$ & 0.104 \\
\hline CRP (mg/dl) & $8.5(3.7-15.1)$ & $9.4(4.8-16.0)$ & $7.1(3.7-14.7)$ & 0.364 \\
\hline Procalcitonin, $n=62,(\mathrm{mg} / \mathrm{dl})$ & $1.1(0.4-5.9)$ & $1.7(0.5-6.8)$ & $0.9(0.3-5.6)$ & 0.571 \\
\hline \multicolumn{5}{|l|}{ Indices for disease severity } \\
\hline CURB-65 score & $2(1-2)$ & $2(1-3)$ & $2(1-2)$ & 0.095 \\
\hline CURB-65 score $\geq 3$ & $19(18.0 \%)$ & 7 (29.1\%) & $12(14.8 \%)$ & 0.133 \\
\hline PSI score & $123(105-145)$ & $148(120-181)$ & 118 (99-139) & 0.001 \\
\hline PSI class IV or V & $91(86.6 \%)$ & $23(95.8 \%)$ & $68(83.9 \%)$ & 0.181 \\
\hline \multicolumn{5}{|l|}{ Initial antibiotic therapy } \\
\hline as CAP & $47(44.7 \%)$ & $4(16.6 \%)$ & $43(53.0 \%)$ & 0.002 \\
\hline as HAP & $58(55.2 \%)$ & $20(83.3 \%)$ & $38(46.9 \%)$ & 0.002 \\
\hline Use of Anti-MRSA agents & $7(6.6 \%)$ & $4(16.6 \%)$ & $3(3.7 \%)$ & 0.046 \\
\hline \multicolumn{5}{|l|}{ Clinical outcomes } \\
\hline Use of inappropriate antibiotics & $21(20.0 \%)$ & $15(62.5 \%)$ & $6(7.4 \%)$ & $<0.001$ \\
\hline Change of initial antibiotics & $40(38.0 \%)$ & $13(54.1 \%)$ & $27(33.3 \%)$ & 0.065 \\
\hline Failure of initial antibiotics therapy & $29(27.6 \%)$ & $11(45.8 \%)$ & $18(22.2 \%)$ & 0.254 \\
\hline Duration of antibiotic therapy (days) & $12(10-15)$ & $12(9-22)$ & $12(10-15)$ & 0.401 \\
\hline
\end{tabular}


Table 1 Baseline clinical characteristics and treatment outcomes of patients admitted with hemodialysis-associated pneumonia (Continued)

\begin{tabular}{lllll}
\hline Characteristics & $\begin{array}{l}\text { Overall patients } \\
(n=105)\end{array}$ & $\begin{array}{l}\text { MDR pathogens group } \\
(n=24)\end{array}$ & $\begin{array}{l}\text { Non-MDR pathogens group } \\
(n=81)\end{array}$ & $P$ Value \\
\hline Length of hospital stay (days) & $11(7-17)$ & $14(9-25)$ & $11(7-16)$ & 0.093 \\
Pneumonia-related mortality rate & $8(7.6 \%)$ & $6(25.0 \%)$ & $2(2.4 \%)$ & 0.002 \\
Hospital mortality rate & $11(10.4 \%)$ & $6(25.0 \%)$ & $5(6.1 \%)$ & 0.016 \\
\hline
\end{tabular}

Data are presented as median (interquartile range) or number (\%)

MDR multidrug-resistant, HCAP healthcare-associated pneumonia, HDAP hemodialysis-associated pneumonia, NHAP nursing home-acquired pneumonia, ICU intensive care unit, WBC white blood cell, CRP C-reactive protein, CAP community-acquired pneumonia, MRSA methicillin-resistant Staphylococcus aureus, CURB-65 Confusion, Urea, Respiratory rate, Blood pressure, Age $\geq 65$, PSI Pneumonia Severity Index

allowed for overlap

negative bacteria $P$. aeruginosa, A. baumanii, ESBLproducing $K$. pneumoniae were $6.6,5.7$, and $2.8 \%$, respectively.

\section{Predictive factors associated with occurrence of MDR pathogens}

Table 3 shows multivariate logistic regression analyses of the four risk factors for MDR pathogens: tube feeding, recent hospitalization, NHAP, and PSI score. Recent

Table 2 Microorganisms identified in patients admitted with hemodialysis-associated pneumonia

\begin{tabular}{|c|c|}
\hline Microorganisms $^{\mathrm{a}}$ & No. of patients (\%) \\
\hline $\begin{array}{l}\text { Identified microorganisms } \\
\text { Gram-positive bacteria }\end{array}$ & $53(50.4 \%)$ \\
\hline Streptococcus pneumoniae & $10(9.5 \%)$ \\
\hline Staphylococcus aureus & $17(16.1 \%)$ \\
\hline MSSA & $7(6.6 \%)$ \\
\hline MRSA & $10(9.5 \%)$ \\
\hline \multicolumn{2}{|l|}{ Gram-negative bacteria } \\
\hline Pseudomonas aeruginosa & $7(6.6 \%)$ \\
\hline Haemophilus influenza & $0(0 \%)$ \\
\hline Klebsiella pneumoniae & $11(10.4 \%)$ \\
\hline ESBL $(+)$ & $3(2.8 \%)$ \\
\hline ESBL (-) & $8(7.6 \%)$ \\
\hline Acinetobacter baumannii & $6(5.7 \%)$ \\
\hline Stenotrophomonas maltophilia & $1(0.9 \%)$ \\
\hline Mycoplasma pneumonia & $2(1.9 \%)$ \\
\hline Other gram-negative species ${ }^{c}$ & $5(4.7 \%)$ \\
\hline Polymicrobial pathogens & $8(7.6 \%)$ \\
\hline Multidrug-resistant pathogens ${ }^{d}$ & $24(22.8 \%)$ \\
\hline
\end{tabular}

Data are presented as number (\%)

HDAP: hemodialysis-associated pneumonia; MSSA: methicillin-sensitive

Staphylococcus aureus; MRSA: methicillin-resistant Staphylococcus aureus

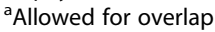

${ }^{b}$ One or more pathogens may be listed

'Other gram-negative species include Escherichia coli, Enterobacter species, Serratia marcescens, and Legionella pneumophilia

${ }^{d}$ Multidrug-resistant pathogens include Methicillin-resistant Staphylococcus aureus (MRSA), Pseudomonas species, Acinetobacter species, Stenotrophomonas maltophilia, and extended-spectrum $\beta$-lactamase

(ESBL)-producing Enterobacteriaceae hospitalization and PSI score was independently associated with the isolation of MDR pathogens in HDAP patients (adjusted OR: 2.951, 95\% CI: 1.022-8.518, $p=0.045$ and adjusted OR: 1.023 , 95\% CI: $1.005-1.041$, $p=0.011$, respectively). ROC curve analysis was used to assess optimal cutoff values for PSI score. The maximum sum of sensitivity and specificity was 147 for PSI (sensitivity $54.1 \%$, specificity $85.1 \%$, positive predictive value $52.0 \%$, and negative predictive value $86.2 \%$, Fig. 2 ).

\section{Proposed decision support tool for prediction of MDR pathogens}

Based on the multivariate logistic regression analysis of the association with occurrence of MDR pathogens in patients with HDAP, PSI score > 147 and recent hospitalization were considered as predictive MDR risk factors. We created a decision support tool to predict MDR pathogens. Patients divided into low (without any risk factors)-, intermediate (with only one risk factor) -, and high (with both two risk factors)-risk strata based on two predictive MDR risk factors.

The ROC curves for prediction tool and PSI score are shown in Fig. 2 The prediction tool had a higher discriminatory power to identify MDR pathogen infection than PSI score, although there was no statistically significant difference $(p=0.228)$. The area under the curve (AUC) of the prediction tool (AUC: 0.764, 95\% CI: 0.652-0.875, $p<0.001$ ) tended to be greater than that of the PSI score (AUC: 0.718, 95\% CI: 0.593-0.842, $p=0.001)(p=0.236)$. The optimal cutoff for the prediction tool was 1 (sensitivity $83.3 \%$, specificity $59.3 \%$,

Table 3 Multivariate logistic regression analysis for predictive factors associated with multidrug-resistant pathogens in patients admitted with hemodialysis-associated pneumonia

\begin{tabular}{lll}
\hline Risk factors & Odds ratio (95\% confidence interval) & $P$ Value \\
\hline Tube feeding & $2.229(0.459-10.819)$ & 0.320 \\
Recent hospitalization & $2.951(1.022-8.518)$ & 0.045 \\
NHAP & $3.535(0.823-15.183)$ & 0.090 \\
PSI score & $1.023(1.005-1.041)$ & 0.011 \\
\hline
\end{tabular}

NHAP nursing home-acquired pneumonia, PSI Pneumonia Severity Index 


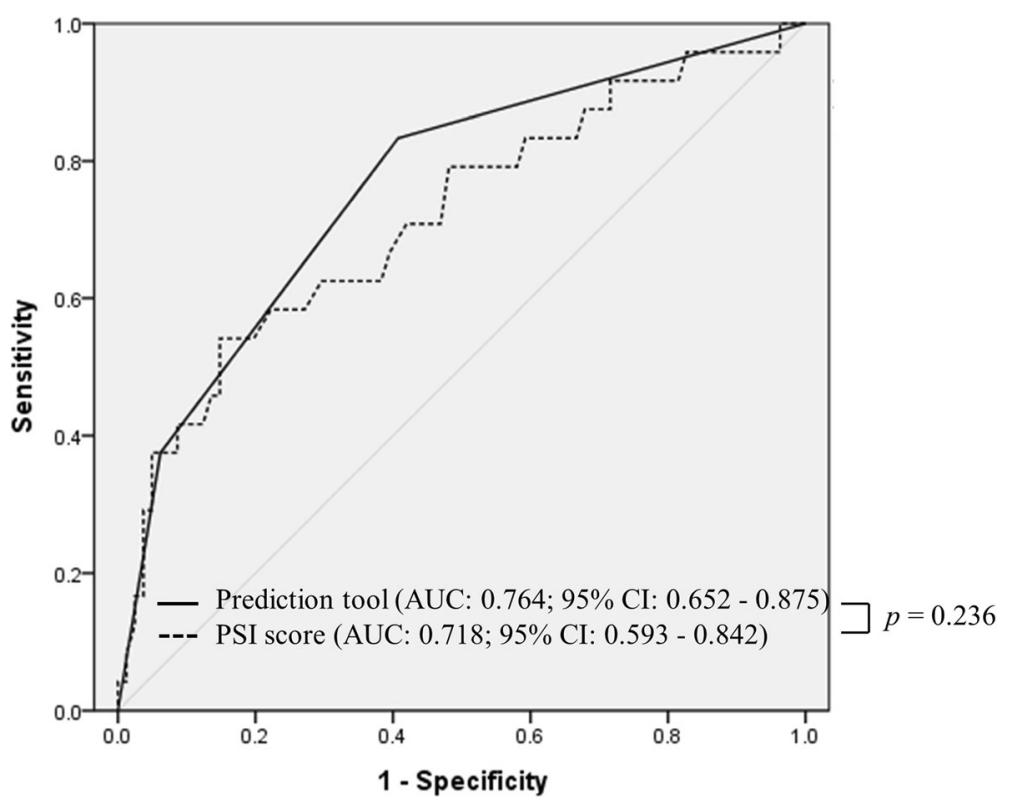

Fig. 2 Comparison of receiver-operating characteristic (ROC) curves between the proposed tool and PSI score to predict infection with multidrug-resistant pathogens. PSI, pneumonia severity index

positive predictive value $37.7 \%$, and negative predictive value 92.3\%). According to the risk stratification based on number of MDR risk variables, the prevalence of MDR pathogens was 7.6, 28.2 and $64.2 \%$, respectively ( $\mathrm{p}<0.001$ for trend, Table 4 and Fig. 3).

\section{Discussion}

The current study revealed that $22.8 \%$ of hospitalized patients with HDAP had MDR pathogens. We also demonstrated that the occurrence of MDR pathogens was significantly associated with recent hospitalization within 3 months and PSI score more than 147. On the basis of these findings, we proposed a simple predictive tool to determine the risk of infection with MDR pathogens in HDAP using the number of risk factors. As the number of risk factors increased, the prevalence of infection with MDR pathogens also increased (low - 7.6\%, intermediate - 28.2\% and high $-64.2 \%$, respectively; $p<0.001$ for trend). Overall, this model had moderate predictive value, as demonstrated by the ROC curve (AUC: 0.764, 95\% CI: 0.652-0.875). To our knowledge, this is the first study that proposes evidence based tool to predict infection with MDR pathogens among HDAP patients.
Previous studies have demonstrated that the isolation rate of MDR pathogens shows interregional differences in HCAP patients [5]. Also, HCAP consists of very heterogeneous subgroups [4], and there is little evidence that all criteria for HCAP convey similar risks for infection with MDR pathogens. This contributed to the removal of the concept of HCAP in the new 2016 ATS/IDSA guidelines for management of HAP and ventilator-associated pneumonia [6]. But, the concept of HCAP as a separate clinical entity would be still valid, because frequent interactions with the healthcare system can have potential risk for MDR pathogens [6]. Discordant results about the isolated rate of MDR pathogens among previous studies may be caused by the fact that the concept of HCAP includes various criteria for heterogeneous conditions [4], which did not have similar risks for infection with MDR pathogens [19]. Among the category of HCAP, only NHAP have been studied considerably, and MDR pathogens were not frequently isolated in these patients [20-25]. In contrast, the previous studies on HCAP included a relatively small proportion of $2.5 \sim 10.4 \%$ of HDAP patients [7, 13, 26-29]. Therefore, it is not known whether it is desirable to actively apply the guidelineconcordant treatment to all patients with HDAP [4].

Table 4 Proposed prediction tools for multidrug-resistant pathogens in patients admitted with hemodialysis-associated pneumonia

\begin{tabular}{|c|c|c|c|}
\hline Risk of MDR pathogens & Predictive factors & No. of patients & $\begin{array}{l}\text { No. (\%) of patients isolated } \\
\text { with MDR pathogens }\end{array}$ \\
\hline Low & PSI score $\leq 147$ and no recent hospitalization & 52 & $4(7.6 \%)$ \\
\hline Moderate & PSI score > 147 or recent hospitalization & 39 & $11(28.2 \%)$ \\
\hline High & PSI score > 147 and recent hospitalization & 14 & $9(64.2 \%)$ \\
\hline
\end{tabular}

MDR multidrug-resistant, $P S /$ Pneumonia: Severity Index 


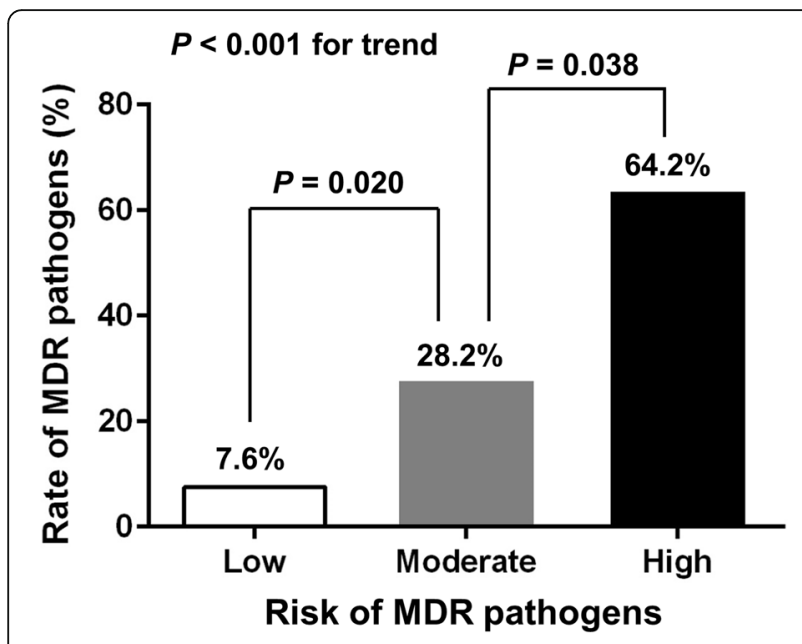

Fig. 3 The probability of MDR pathogens stratified by risk using the prediction tool. MDR, multidrug-resistant

There are few studies focusing on the actual risk of MDR pneumonia in HDAP patients, resulting in a lack of microbiologic data on HDAP as a different category of HCAP. Although most HD patients live outside of hospitals, they manifest various degrees of immunodeficiency, regularly visit the hospital, and receive ongoing healthcare more often than non-HD patients. Compared to the general population, these characteristics of $\mathrm{HD}$ patients may contribute to the high incidence of MDR pathogens, which is not related to HD itself. Therefore, there is a question regarding whether all patients with HDAP should receive antibiotic therapy against MDR pathogens [30, 31].

We found two large cohort studies using the USRDS registry for clinical epidemiology of HDAP patients and four retrospective studies applying to the concept of HDAP [1, 2, 7, 30-32]. Unfortunately, in large cohort studies, the microorganisms in more than $80 \%$ of the patients hospitalized with HDAP could not be identified $[1,2]$. The detection rate of $P$. aeruginosa was about $2 \%$, but the rate of total isolated MDR pathogens was not mentioned in either study $[1,2]$. In addition, four retrospective studies have demonstrated inconsistent MDR pathogen distributions in HDAP patients [7, 30-32]. In line with previous HDAP studies, the rate of isolated MDR pathogens was 5.6 to $35.4 \%$, although microorganisms could not be identified in most patients. The detection rates of MRSA, $P$. aeruginosa, and A. baumanii were $0 \% \sim 27.5 \%$, $1.6 \% \sim 16.7 \%$, and $0 \% \sim 4.2 \%$, respectively $[7,30-32]$. In the present study, MDR pathogens were identified in $22.8 \%$ of cases; of these, the most frequent microorganisms were MRSA (9.5\%), followed by P. aeruginosa (6.6\%), A. baumanii (5.7\%). However, the rates of isolated MDR pathogens in previous studies and this study revealed a variable incidence range [7, 30-32].
Although we cannot offer satisfactory explanations for these various incidence rates of MDR pathogen distribution, these discordant results may be related to whether the selected populations included the other criteria for HCAP. The studies demonstrating relatively low MDR pathogen infections included patients who only met HDAP criterion without O-HCAP components for HCAP classification [7, 31]. On the contrary, the remaining studies enrolled patients who included the other criteria for HCAP, and reported relatively high incidence of MDR pathogens [30, 32]. In line with this concept, among 61 patients who only met HDAP criterion without the other criteria for HCAP in our study, the isolated rate of MDR pathogens was relatively low (7 patients, $11.4 \%)$. We also demonstrated that presence of O-HCAP components exhibited higher occurrence of MDR pathogens in patients with HDAP. Among the OHCAP category, recent hospitalization and NHAP were more frequently observed in the MDR pathogens group. Especially, recent hospitalization was independently associated with the isolation of MDR pathogens in multivariable analysis. Therefore, our study found that MDR pathogen infection in HDAP could be associated with recent hospitalization, rather than HD status itself, similar to previous studies $[19,26]$. The present study revealed that PSI score than 147 was also significantly associated with HDAP caused by MDR pathogens. Furthermore, the proposed prediction tool using these two risk factors showed a moderate discriminatory power for risk stratification for an infection with MDR pathogens in HDAP patients. Therefore, our findings could be helpful in physicians' decisions to select HDAP patients who need to be treated for MDR pathogens.

The present study has several limitations. Firstly, the main limitation of this study is retrospective design. Because the effect of missing data in the results is unknown, our study may be vulnerable to selection bias. And a small sample size did not allow us to draw a robust conclusion. We were unable to enroll a large number of HDAP patients despite the fact that they were collected from three centers over 5 years. Larger studies are needed to validate our results and to strengthen the power to identify risk factors of MDR pathogens. Secondly, the microbiological etiology could be identified in only about $50 \%$ of enrolled patients. Possible reasons for low detections would include an inability to collect lower respiratory tract specimens, prior antibiotic use before specimen collection, insensitive diagnostic tests for known pathogens, a lack of testing for other recognized pathogens such as coxiella, unknown pathogens, and possible noninfectious causes such as aspiration pneumonitis [33]. Thirdly, median age of the patients is 71 years in the present study. Aging is associated with declines in adaptive and innate immunity [34]. Infections occur more frequently in the elderly, and the age-related remodeling of the immune system plays 
any role in the development of HDAP or HDAP with MDR pathogens [34]. Although age was not associated with the isolation of MDR pathogens among HDAP patients in our study, the results might be biased towards the old age population. Finally, the analysis for distribution of MDR pathogens included entire study populations with culturenegative pneumonia. Thus, we may have skewed our findings in that the true incidence of MDR pathogens could have been underestimated. In this study, we founded two independent risk factors for occurrence of MDR pathogens in patients with HDAP. And the prevalence of MDR pathogens was $<10 \%$ in patients without one of these risk factors. If validated in subsequent multicenter studies, this prediction rule could potentially assist clinicians who are deciding on whether to administer anti-MRSA or anti-pseudomonal therapy to patients with HDAP.

\section{Conclusion}

This multicenter, retrospective, observational study offers the findings of the clinical epidemiology, microbiology, and predictive factors of MDR pathogens in patients with HDAP. The HDAP concept itself as an HCAP has limited value in selecting patients harboring MDR pathogens. It could be necessary to stratify the patients with regard to risk factors in order to properly identify infection with MDR pathogens. Although large-scale prospective studies are needed to confirm our results, our findings would be helpful for physicians' decisions to select HDAP patients harboring MDR pathogens.

\begin{abstract}
Abbreviations
A. baumannii: Acinetobacter baumannii; ATS/IDSA: American Thoracic Society/ Infectious Diseases Society of America; CAP: community-acquired pneumonia; CRP: C-reactive protein; CURB-65: Confusion, Urea, Respiratory rate, Blood pressure, Age $\geq 65$; ESBL: Extended-spectrum b-lactamase; HAP: Hospital-acquired pneumonia; HCAP: Healthcare-associated pneumonia; HD: Hemodialysis; HDAP: Hemodialysis-associated pneumonia; ICU: Intensive care unit; $K$. pneumoniae: Klebsiella pneumoniae; MDR: Multidrug-resistant; MRSA: Methicillinresistant Staphylococcus aureus; NHAP: Nursing home-acquired pneumonia; OHCAP: The HCAP other than HDAP; $P$. aeruginosa: Pseudomonas aeruginosa; PSI: Pneumonia Severity Index; S. aureus: Staphylococcus aureus; S. pneumoniae: Streptococcus pneumoniae; USRDS: The United States Renal Dada System; WBC: White blood cell
\end{abstract}

\section{Acknowledgements}

Nil.

\section{Funding}

This study was funded by a research grant from Chong Kun Dang Corp.

\section{Availability of data and materials}

All the data supporting our findings are contained within the manuscript.

\section{Authors' contributions}

J-US and HKP contributed to the study design, data acquisition, and writing of the manuscript. HKK contributed to data acquisition, and data interpretation. $J \mathrm{~L}$ contributed to the study design, data acquisition, data interpretation, statistical analysis, writing of the manuscript, and is responsible for the study. All authors read and approved the final manuscript.

\section{Ethics approval and consent to participate}

The study protocol was approved by the Ethical Review Committee of Jeju National University Hospital (IRB number, 2015-07-005). Informed consent was waived because of the retrospective nature of the study.

Consent for publication

Not applicable.

\section{Competing interests}

The authors declare that they have no competing interests.

\section{Publisher's Note}

Springer Nature remains neutral with regard to jurisdictional claims in published maps and institutional affiliations.

\section{Author details}

${ }^{1}$ Division of Pulmonary and Critical Care Medicine, Department of Internal Medicine, Kangbuk Samsung Hospital, Sungkyunkwan University School of Medicine, Seoul, South Korea. ${ }^{2}$ Division of Pulmonary and Critical Care Medicine, Department of Internal Medicine, Ilsan Paik Hospital, Inje University College of Medicine, Goyang-si, South Korea. ${ }^{3}$ Department of Internal Medicine, Jeju National University Hospital, Jeju National University School of Medicine, Aran 13 gil 15, Jeju-si, Jeju Special Self-Governing Province 690-767, South Korea.

Received: 30 March 2017 Accepted: 4 October 2017

Published online: 12 October 2017

\section{References}

1. Guo H, Liu J, Collins AJ, Foley RN. Pneumonia in incident dialysis patientsthe United States renal data system. Nephrol Dial Transplant. 2008;23:680-6.

2. Slinin Y, Foley RN, Collins AJ. Clinical epidemiology of pneumonia in hemodialysis patients: the USRDS waves 1, 3, and 4 study. Kidney Int. 2006; 70:1135-41.

3. Vanholder R, Ringoir S. Infectious morbidity and defects of phagocytic function in end-stage renal disease: a review. J Am Soc Nephrol. 1993;3: $1541-54$.

4. American Thoracic Society. Infectious Diseases Society of America. Guidelines for the management of adults with hospital-acquired, ventilatorassociated, and healthcare-associated pneumonia. Am J Respir Crit Care Med. 2005;171:388-416.

5. Chalmers JD, Rother C, Salih W, Ewig S. Healthcare-associated pneumonia does not accurately identify potentially resistant pathogens: a systematic review and meta-analysis. Clin Infect Dis. 2014:58:330-9.

6. Kalil AC, Metersky ML, Klompas M, Muscedere J, Sweeney DA, Palmer LB, et al. Management of Adults with Hospital-Acquired and VentilatorAssociated Pneumonia: 2016 clinical practice guidelines by the Infectious Diseases Society of America and the American Thoracic Society. Clin Infect Dis. 2016;63:e61-e111.

7. Lee JH, Moon JC. Clinical characteristics of patients with hemodialysisassociated pneumonia compared to patients with non-hemodialysis community-onset pneumonia. Respir Med. 2016;111:84-90.

8. Lee YK, Kim K, Kim DJ. Current status and standards for establishment of hemodialysis units in Korea. Korean J Intern Med. 2013;28:274-84.

9. Korean Standard Statistical Classification. The standard of classification of disease and cause of death [Internet]. Daejeon, Korea; Korean Standard Statistical Classification; 2016 [cited at 2017 Aug 11]. Available from: https:// kssc.kostat.go.kr:8443/ksscNew_web/link.do?gubun=AQ5004.

10. Lim WS, van der Eerden MM, Laing R, Boersma WG, Karalus N, Town Gl, et al. Defining community acquired pneumonia severity on presentation to hospital: an international derivation and validation study. Thorax. 2003;58:377-82

11. Fine $M J$, Auble TE, Yealy DM, Hanusa BH, Weissfeld LA, Singer DE, et al. A prediction rule to identify low-risk patients with community-acquired pneumonia. New Engl J Med. 1997;336:243-50.

12. Mandell LA, Wunderink RG, Anzueto A, Bartlett JG, Campbell GD, Dean NC, et al. Infectious Diseases Society of America/American Thoracic Society consensus guidelines on the management of community-acquired pneumonia in adults. Clin Infect Dis. 2007;44(Suppl 2):S27-72. 
13. Micek ST, Kollef KE, Reichley RM, Roubinian N, Kollef MH. Health careassociated pneumonia and community-acquired pneumonia: a singlecenter experience. Antimicrob Agents Chemother. 2007;51:3568-73.

14. Maruyama T, Fujisawa T, Okuno M, Toyoshima H, Tsutsui K, Maeda H, et al. A new strategy for healthcare-associated pneumonia: a 2-year prospective multicenter cohort study using risk factors for multidrug-resistant pathogens to select initial empiric therapy. Clin Infect Dis. 2013;57:1373-83.

15. Geckler RW, Gremillion DH, McAllister CK, Ellenbogen C. Microscopic and bacteriological comparison of paired sputa and transtracheal aspirates. J Clin Microbiol. 1977;6:396-9.

16. Clinical and Laboratory Standards Institute: Methods for Dilution Antimicrobial Susceptiblity Tests for Bacteria That Grow Aerobically; Approved StandardEighth Edition. Clin Lab Stand Inst 2008;29:M07-A08.

17. Hanley JA, McNeil BJ. The meaning and use of the area under a receiver operating characteristic (ROC) curve. Radiology. 1982;143:29-36.

18. Bewick V, Cheek L, Ball J. Statistics review 13: receiver operating characteristic curves. Crit Care. 2004;8:508-12.

19. Shorr AF, Zilberberg MD, Micek ST, Kollef MH. Prediction of infection due to antibiotic-resistant bacteria by select risk factors for health care-associated pneumonia. Arch Intern Med. 2008;168:2205-10.

20. Ewig S, Klapdor B, Pletz MW, Rohde G, Schutte H, Schaberg T, et al. Nursing home-acquired pneumonia in Germany: an 8-year prospective multicentre study. Thorax. 2012;67:132-8.

21. Ma HM, Wah JL, Woo J. Should nursing home-acquired pneumonia be treated as nosocomial pneumonia? J Am Med Dir Assoc. 2012;13:727-31.

22. Polverino E, Dambrava P, Cilloniz C, Balasso V, Marcos MA, Esquinas C, et al. Nursing home-acquired pneumonia: a 10 year single-centre experience. Thorax. 2010;65:354-9.

23. Nakagawa N, Saito Y, Sasaki M, Tsuda Y, Mochizuki H, Takahashi H. Comparison of clinical profile in elderly patients with nursing and healthcare-associated pneumonia, and those with community-acquired pneumonia. Geriatr Gerontol Int. 2014;14:362-71.

24. Liapikou A, Polverino E, Cilloniz C, Peyrani P, Ramirez J, Menendez R, et al. A worldwide perspective of nursing home-acquired pneumonia compared with community-acquired pneumonia. Respir Care. 2014:59:1078-85.

25. Koh SJ, Lee JH. Clinical characteristics of nursing home-acquired pneumonia in elderly patients admitted to a Korean teaching hospital. Korean J Intern Med. 2015;30:638-47.

26. Jung JY, Park MS, Kim YS, Park BH, Kim SK, Chang J, et al. Healthcareassociated pneumonia among hospitalized patients in a Korean tertiary hospital. BMC Infect Dis. 2011;11:61.

27. Chalmers JD, Taylor JK, Singanayagam A, Fleming GB, Akram AR, Mandal $P$, et al. Epidemiology, antibiotic therapy, and clinical outcomes in health careassociated pneumonia: a UK cohort study. Clin Infect Dis. 2011;53:107-13.

28. Shindo Y, Sato S, Maruyama E, Ohashi T, Ogawa M, Hashimoto N, et al. Health-care-associated pneumonia among hospitalized patients in a Japanese community hospital. Chest. 2009;135:633-40.

29. Zilberberg MD, Shorr AF, Micek ST, Mody SH, Kollef MH. Antimicrobial therapy escalation and hospital mortality among patients with health-careassociated pneumonia: a single-center experience. Chest. 2008;134:963-8.

30. Kawasaki S, Aoki N, Kikuchi H, Nakayama H, Saito N, Shimada H, et al. Clinical and microbiological evaluation of hemodialysis-associated pneumonia (HDAP): should HDAP be included in healthcare-associated pneumonia? J Infect Chemother. 2011;17:640-5.

31. Taylor SP, Taylor BT. Health care-associated pneumonia in haemodialysis patients: clinical outcomes in patients treated with narrow versus broad spectrum antibiotic therapy. Respirology. 2013;18:364-8.

32. Wang PH, Wang HC. Risk factors to predict drug-resistant pathogens in hemodialysis-associated pneumonia. BMC Infect Dis. 2016:16:377.

33. Jain $S$, Self WH, Wunderink RG, Fakhran S, Balk R, Bramley AM, et al. Community-acquired pneumonia requiring hospitalization among U.S adults. N Engl J Med. 2015;373:415-27.

34. Fuentes $\mathrm{E}$, Fuentes $\mathrm{M}$, Alarcón $\mathrm{M}$, Palomo I. Immune system dysfunction in the elderly. An Acad Bras Cienc. 2017;89:285-99.

\section{Submit your next manuscript to BioMed Central and we will help you at every step:}

- We accept pre-submission inquiries

- Our selector tool helps you to find the most relevant journal

- We provide round the clock customer support

- Convenient online submission

- Thorough peer review

- Inclusion in PubMed and all major indexing services

- Maximum visibility for your research

Submit your manuscript at www.biomedcentral.com/submit
Biomed Central 